\title{
Spotlight on Israeli science products
}

As an accompaniment to this week's special feature on Science in Israel, the spotlight falls on a collection of Israeli diagnostics, drugs, research reagents, and medical products.

INTERNATIONAL Diagnostic Laboratories (IDL), located at the centre of the Jerusalem Science-Based Industrial Park, is aiming to attract a portion of the international lipid diagnostic market with several new kits (Reader Service No. 100). Earlier this year, IDL entered the US and European markets with immunoturbimetric kits for the measurement of apolipoproteins A-I and $\mathrm{B}$. As the two principal protein components of HDL and LDL, the presence of these two apolipoproteins serves as an early detection signal for the diagnosis of cardiovascular disease. IDL calibrates its apolipoprotein kit reagents against a $\mathrm{CDC}$ international candidate reference serum, and uses high-titre, pooled polyclonal goat anti-human antisera. As a companion to its kits, IDL offers a dedicated bench-top instrument that can read and store calibration curves, and deliver absolute values in $\mathrm{mg} \mathrm{dl^{-1 }}$ for samples prepared any time during the day. Kits for the measurement of apolipoproteins $\mathrm{C}$ and $\mathrm{E}$, as well as classical enzyme assays for cholesterol and triglycerides, are currently in the IDL research pipeline.

Gelman Sciences Technology, Inc., in Rehovot, is involved in the production of medical nonwoven sterile barriers for use in bandages, gowns, disposable bed sheets, and baby and adult incontinent products (Reader Service No. 101). The

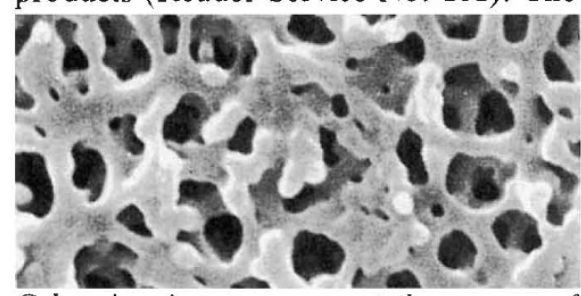

Gelman's micropores prevent the passage of germs while allowing air to circulate freely.

nonwoven barriers have micropores ranging in size from 0.1 to $0.5 \mu \mathrm{m}$, and Gelman reports that they retain 99.999 per cent of bacteria while allowing air and water vapour to pass through freely. The nonwovens are prepared by polymerizing small molecules bearing highly reactive acrylate functional groups by exposing them to ultraviolet light. The result is a "breathable" autoclavable sterile barrier that can be mass-produced with relative ease: Gelman reports its pilot plant can turn out the material at 150 feet per minute with a UV light source, and 300 feet per minute using an electron beam apparatus.

Orgenics Ltd, in Yavne, Israel, is also involved in the development of health- care diagnostics, and has kits for both human and veterinary use (Reader Service No. 102). Orgenics' line of self-contained ImmunoComb kits consist of a plastic comb and development plate. ImmunoComb kits have been produced for the diagnosis of Mycoplasma gallisepticum and $M$. synoviae infections in poultry, and $M$. pneumoniae, Coronaviruses and $\mathrm{MHV}$ in mice and rats. Human diagnostics include tests for the detection of $\operatorname{IgE}$, and Rubella and Chlamydia antibodies. Orgenics is working on a human DNA probe kit for detecting $M$. pneumoniae directly from patient sputum samples, that uses a DNA labelling system involving sulphonation of the DNA followed by recognition by monoclonal antibody detected with a chromogenic reaction with a second enzymelabelled antibody.

The competition of high titres of immune IgG with IgM for the same antigenic sites can produce false negative results in tests for IgM. Similarly, rheumatoid factor can also produce false results in the presence of IgG. To overcome these problems, Savyon Diagnostics Ltd in Ramat Gan has an IgG/rf stripping solution for the treatment of serum before conducting IgM assays (Reader Service No 103). Savyon's stripping solution is incorporated in its IPAzyme True-IgM series of tests for the detection of IgM specific to Chlamydia trachomatis and EEV.

A new brochure, entitled Immunology Research News, is now available from International Bio-Technologies Ltd, in Jerusalem (Reader Service No. 104). The free eight-page brochure outlines the uses of IBT's extracellular matrix (ECM) coated labware in immunological applications. IBT says the ECM labware is suited for research on the extravasation of neutrophils, T-lymphocytes and macrophages, autoimmune diseases, immune system interactions with malignant cells, and proliferation and differentiation of hematopoietic cells. The brochure also touches on the uses of radioactively labelled ECM coated dishes in matrix degradation studies.

ExtrAvidin is BioMakor's answer to nonspecific binding problems (Reader Service No. 105). The Rehovot-based company's ExtrAvidin, developed in conjunction with the Weizmann Institute of Science, combines the high specific activity and sensitivity of avidin with the low background staining of streptavidin. BioMakor says ExtrAvidin binds biotin with the high affinity of egg white avidin $\left(K_{\mathrm{D}}=\right.$ $10^{-15}$ ) but without the nonspecific binding, such as the staining of mast cells, exhibited by egg white avidin at physiological $p \mathrm{H}$. ExtrAvidin comes unlabelled, or conjugated with alkaline phosphatase, peroxidase, FITC and TRITC.

FRONE is the name of InterPharm Laboratories Ltd's native beta interferon product, available as a topical cream or as an intralesional injection (Reader Service No. 106). FRONE has been approved in

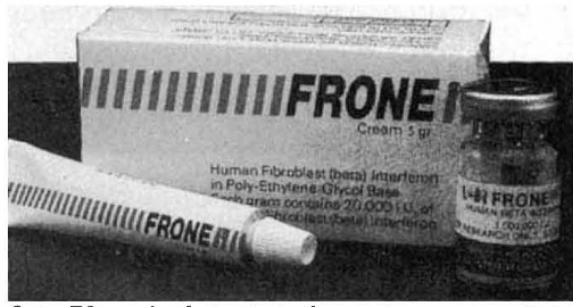

InterPharm's beta interferon is approved in some countries for the treatment of herpes.

Argentina, Italy and Israel for the treatment of Herpes virus infections. InterPharm claims that clinical trials have shown that FRONE reduces or eliminates herpes symptoms, prolongs the interval between attacks, and reduces the severity of subsequent attacks. The company, in Ness-Ziona, plans to concentrate next on developing recombinant beta- 1 interferon, and on making alpha $\mathrm{C}$ and gamma interferon.

Bio-Technology General, with research headquarters in Rehovot, is involved in a range of biotechnological product developments (Reader Service No. 107). BTG has a non-methionyl form of human growth hormone that is nearly ready to enter the market. BTG announced last month that its recombinant superoxide dismutase (SOD) has entered Phase II human clinical trials in the US, to ascertain its efficacy in preventing reperfusion damage associated with kidney transplants. SOD has shown promise in animal trials in mopping up the oxygenfree radicals that occur in tissues and the bloodstream when the supply of oxygen is cut off, and that cause tissue damage upon reperfusion. Currently, BTG sells recombinant hyaluronic acid as a moisturizer/ lubricant for use in cosmetics, soaps and other personal care products.

These notes are compiled by Carol Ezzell from information provided by the manufacturers. To obtain further details about these products, use the reader service card bound inside the journal. 